\title{
Robust Satellite Navigation by Means of a Spherical Cap Conformal Antenna Array
}

\author{
Kazeem A. Yinusa, Emilio Pérez Marcos and Stefano Caizzone \\ German Aerospace Center (DLR) \\ Institute of Communications and Navigation \\ Oberpfaffenhofen, Wessling 82234, Germany \\ Email: kazeem.yinusa@dlr.de
}

\begin{abstract}
This contribution presents the design and performance of a dual-band spherical cap adaptive antenna array for safety-critical satellite navigation. The array consists of seven planar elements, flush-mounted in a spherical cap holder. Each element of the array is resonant at dual frequencies with adequate bandwidth to cover the GPS L1/L5 and Galileo E1/E5a/b frequency bands. The interference mitigation and beamforming performance of the array is demonstrated by means of an adaptive beamforming algorithm. The results show a strong potential of the antenna for applications where continuous system availability is essential.

Index Terms-Conformal Antennas, Antenna Arrays, GNSS, Galileo
\end{abstract}

\section{INTRODUCTION}

Global navigation satellite systems (GNSS) are an important part of modern technological infrastructure. They provide the essential timing services that are needed, for instance, in synchronous telecommunication systems and financial services. GNSS positioning service is invaluable in several sectors, including security, maritime and aviation. For some of these applications, the accuracy requirements are stringent e.g. in aviation approach and landing scenario utilizing satellite navigation signals. Moreover, certain threats such as electromagnetic interference from jammers and spoofers are to be foreseen and counteracted in safety-critical applications.

This paper presents some of the activities at the German Aerospace Center (DLR) aimed at developing more robust navigation receivers. In particular, this paper presents a dualband (E5a,b and E1) antenna array with seven elements arranged on a spherical cap. The shape of the array enables it to provide a fairly constant gain and low axial ratio over the whole upper hemisphere. The paper also presents the array GNSS receiver developed by researchers at DLR.

\section{Dual-Band Antenna Design}

The array consists of seven antenna elements arranged on a spherical cap. Each antenna element utilizes a stacked-patch configuration to achieve resonance at the Galileo E5a/b and E1 frequency bands. The stack-up and dimensions of the single element are shown in Fig. 1. Circular polarization is obtained by combining the two feeds of the antenna with a quadrature phase shift by means of a commercial off-the-shelf hybrid combiner. The single elements are arranged to form an array on a spherical cap as shown Fig. 2. One reference

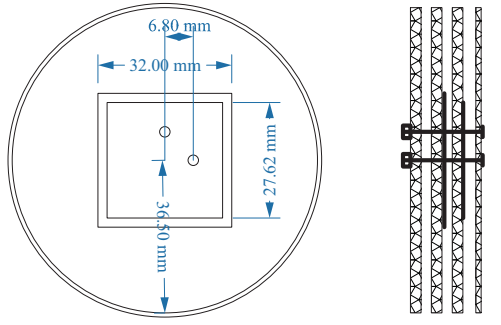

Fig. 1. Layout of the single antenna element. The upper dielectric layer has a thickness of $1.27 \mathrm{~mm}$ and the remaining three layers have a thickness of $2.54 \mathrm{~mm}$. The utilized material has a dielectric constant of 10.2.

element is placed on top of the spherical cap and the other six elements are arranged on a ring below the top element in such a way that each element of the array is radially directed. The lower elements of the array are tilted at an angle of $60^{\circ}$ from zenith and the equitorial diameter of the array is about $24 \mathrm{~cm}$. To accommodate for the larger bandwidth requirement of the E5 frequency band ( $50 \mathrm{MHz})$ compared to the $(32 \mathrm{MHz})$ bandwidth of the L1 band, a thicker substrate layer is utilized below the lower patch. In order to ensure optimum array

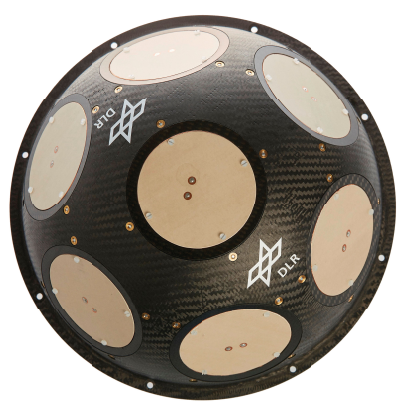

Fig. 2. The manufactured conformal array.

performance, for instance, to form deep nulls in the direction of interferers, the antenna array should be calibrated. The calibration should be performed for the whole signal paths of all antenna elements, including all electronic components, cables and analog-to-digital converters of a digital receiver. Calibration of the presented array is achieved by means of a broadband online calibration network. The calibration network 
divides the calibration signal from the receiver to eight outputs with equal amplitude and phase. Each output port of the calibration network is coupled to a corresponding antenna output with the extra calibration network output terminated with a $50 \Omega$ resistor. The calibration signal then travels through the whole signal paths behind each of the array elements to a digital receiver and enables permanent online calibration. A detailed presentation of the antenna design and calibration network is provided in [1].

\section{Antenna Measurement Results}

The manufactured array was measured in a near-field measurement chamber equipped with a spherical positioner. Each of the seven right hand circular polarization (RHCP) ports was measured while the remaining antenna ports in the array are terminated by $50 \Omega$ resistors. The obtained embedded farfield patterns are shown in Fig. 3 for E5 center frequency of $1.189 \mathrm{GHz}$ with similar results obtained for $\mathrm{E} 1$ center frequency of $1.575 \mathrm{GHz}$. It can be observed that the array provides a relatively good coverage of the upper hemisphere, a performance that can be credited to the hemispherical shape of the array.

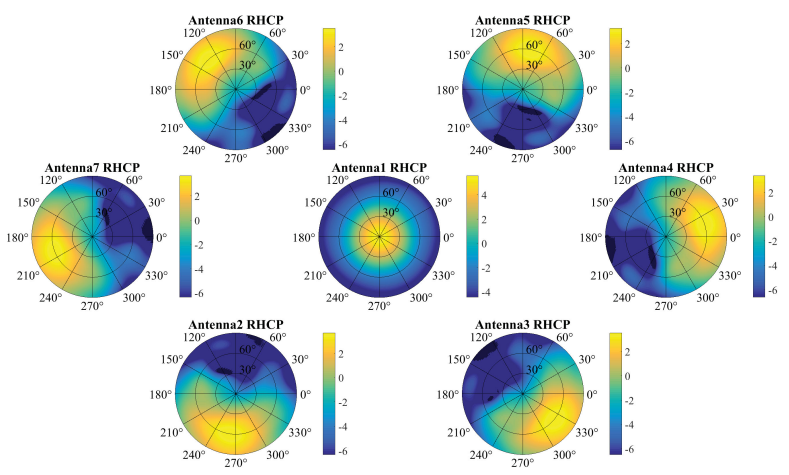

Fig. 3. Embedded RHCP gain patterns of the antenna elements at $1.189 \mathrm{GHz}$ in dBic.

\section{ARRAY RECEIVER}

The block diagram of the array GNSS receiver is displayed in Fig. 4. The propagating radio frequency (RF) signal is received by the antenna and down-converted to an intermediate frequency by the seven dual-band (E5/E1) RF front-ends. In the presence of interference, the receiver is able to place a null towards interference by means of a blind nulling algorithm. The navigation signal from up to sixteen space vehicles (SV) are acquired and continuously tracked by the pseudorandom noise (PRN) correlation blocks which are implemented in field-programmable gate array. The receiver utilizes a digital blind beamforming algorithm which enables the formation of several simultaneous beams in order to optimize the estimated carrier to noise ratio $\left(C / N_{o}\right)$ of the tracked satellites. An instance of such beam forming toward a target satellite can be seen in Fig. 5 where the beam of the antenna array is steered towards the Galileo satellite with PRN code 9 and GPS

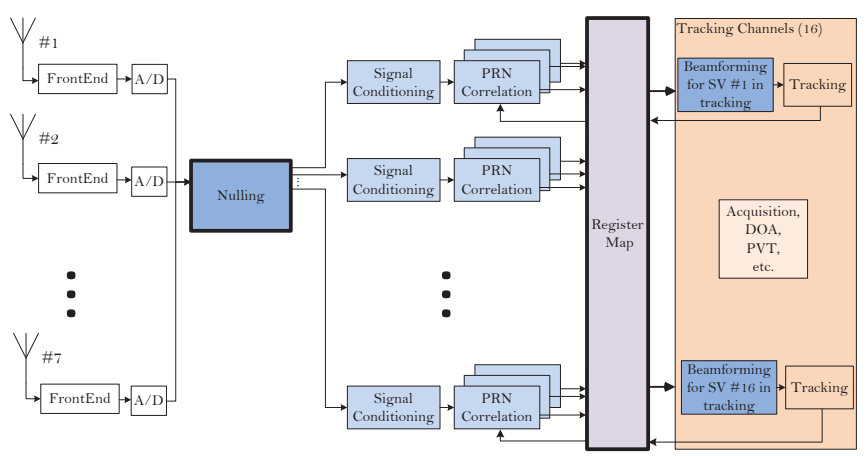

Fig. 4. Architecture of an array GNSS receiver.

satellite with PRN code 15. The implemented beamforming algorithm is adaptive to the interference scenario such that the array pattern is not steered towards a predetermined direction but formed to maximize the $C / N_{o}$. Reference [2] provides more details about a modified version of the receiver for maritime applications.

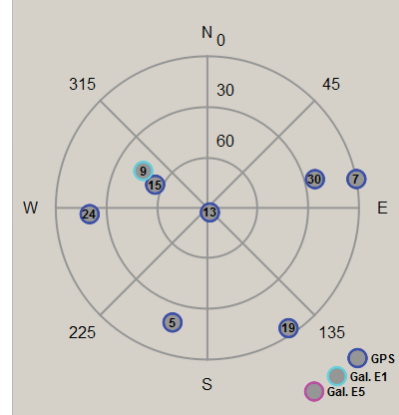

(a) Skyplot of tracked satellites

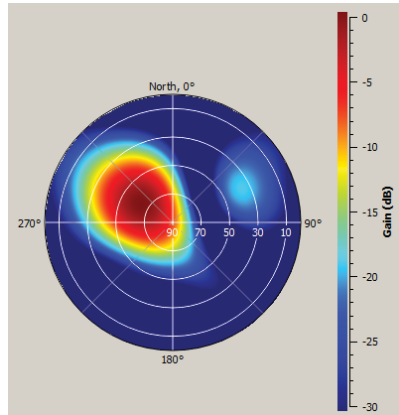

(b) Beamformed pattern
Fig. 5. Digital beamforming: The skyplot in the left shows the constellation of satellites that are being tracked at an instance of time and the figure in the right shows the normalized array beamforming pattern towards the satellite with PRN 9.

\section{Summary}

An hemispherical dual-band conformal array and a robust array receiver for safety-critical GNSS applications was presented. The shape of the array allows it to provide a relatively good coverage of the whole upper hemisphere. The antenna array and the accompanying array signal processing modules allow the receiver to perform interference/spoofing detection and mitigation and to ensure continuous system availability even in presence of interference.

\section{REFERENCES}

[1] K. A. Yinusa, L. A. Greda, and A. Dreher, "A conformal multi-frequency antenna array for safety-of-life satellite navigation," European Microwave Week, Nuremberg, Germany, Oct. 2017.

[2] E. Pérez Marcos, S. Caizzone, A. Konovaltsev, K. A. Yinusa, W. Elmarissi, M. Cuntz, and M. Meuer, "Interference awareness and characterization for gnss maritime applications," IEEE/Institute of Navigation (ION) Positioning, Location and Navigation Symposium (PLANS), Apr. 2018 . 